\title{
EXISTENCE OF POSITIVE SOLUTIONS OF A NONLINEAR DIFFERENTIAL EQUATION WITH n DELAYS
}

The equation $\dot{y}(t)=-(a+b(t)) \prod_{t=1}^{n} y^{\alpha_{i}}\left(t-\tau_{i}\right)$ is considered where $n$ is a positive integer, a, $\tau_{i}$ and $\alpha_{i}, i=1,2, \ldots, n$ are positive constants and conditions on function $b$ are formulated such that the considered equation has positive solutions when $t \rightarrow \infty$. The equation is studied under assumptions

$$
a<1 /\left(e \sum_{i=1}^{n} \alpha_{i} \tau_{i}\right), \sum_{i=1}^{n} \alpha_{i}=1
$$

Estimation of positive solutions is given as well. The proof is based on the retract technique.

Keywords: Nonlinear differential equation with delays, positive solutions, transcendental equation, asymptotic behavior.

\section{Preliminaries}

New mathematical models which involve differential equations with delay continue to arise with increasing frequency in the modeling of diverse phenomena in physics, biology, ecology, and physiology. Often such equations describe various processes appearing in practice more realistic than differential equations without delays. Let us consider a motivation example: Example (Mixing of liquids) [1]

Consider a tank containing $l$ liters of sugar water solution. Fresh water flows in at the top of the tank at a rate of $m$ liters per minute. The water solution in the tank is continually stirred, and the mixed solution flows out through a hole at the bottom, also at the rate of $m$ liters per minute [Fig. 1]. Determine the amount of sugar in the water solution in the tank at time $t$.

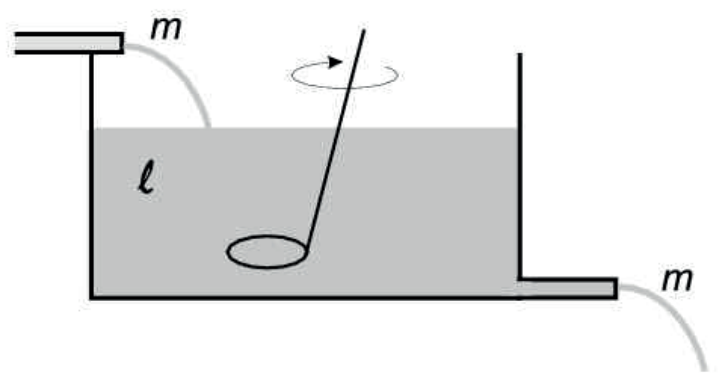

Fig 1 Mixing of liquids
Solution. Let $y(t)$ be the amount (in kilograms) of sugar in the solution in the tank at time $t$. If we assume continual, instantaneous, perfect mixing throughout the tank, then the solution leaving the tank contains $y(t) / l$ kilograms of sugar per liter, and hence the rate of change of the amount of sugar in the solution at time $t$ is proportional to $m y(t) / l$. Therefore we can compose an ordinary differential equation describing the change of the amount of the sugar in the water solution in the tank at the time $t$ :

$$
y^{\prime}(t)=-\frac{m}{l} y(t)
$$

and

$$
y\left(t_{0}\right)=y_{0}
$$

where $y_{0}$ is the amount (in kilograms) of sugar in the solution in the tank at the initial time $t_{0}$. It is easy to see that the solution of (1) is given by the formula

$$
y(t)=y_{0} \exp \left\lceil-\frac{m}{l}\left(t-t_{0}\right)\right\rceil .
$$

A disadvantage of the given model is following. As mixing cannot occur instantaneously throughout the tank it is more realistic to assume that the concentration of the sugar water solution leaving the tank at time $t$ equals the average concentration

\footnotetext{
* Maria Kudelcikova

Department of Mathematics, Faculty of Humanities, University of Zilina, Slovakia

E-mail: maria.kudelcikova@fhv.uniza.sk
} 
at some earlier instant, say $t-\tau$. We shall assume that $\tau$ is a positive constant (often called a time lag or a time delay). Then the differential equation for becomes a delay differential equation

$$
y^{\prime}(t)=-\frac{m}{l} y(t-\tau)
$$

Moreover, the speed of mixing can depend also on the time and then instead of the equation (2) one can consider the following mathematical model:

$$
y^{\prime}(t)=-\frac{m}{l} y(t-\tau(t))
$$

with a positive bounded function $\tau(t)$.

Models (2) or (3) should give a better description of the decrement process of amount of sugar in the tank than the model (1) without delay. Since in all models (1) - (3) it is assumed (and it is expected) $y(t)>0$ if $t \geq t_{0}$ we can classify all of them as problems on existence of a positive solution on interval $\left[t_{0}, \infty\right)$. In the following part of the paper the problem on existence of positive solutions is discussed for a class of nonlinear equations.

\section{Introduction}

Let us consider the following nonlinear first order differential equation with $n$ delays, more complicated than (3),

$$
\dot{y}(t)=-(a+b(t)) \prod_{i=1}^{n} y^{\alpha_{i}}\left(t-\tau_{i}\right)
$$

where $a, \tau$, and $\alpha_{i}, i=1,2, \ldots, n$ are positive constants,

$$
a<1 /\left(e \sum_{i=1}^{n} \alpha_{i} \tau_{i}\right), \sum_{i=1}^{n} \alpha_{i}=1,
$$

$b:\left[t_{0}-\tau, \infty\right) \rightarrow \mathbb{R}^{+}=(0, \infty)$ is a continuous function, $t_{0} \in R$ and $\tau=\max _{i} \tau_{i}$. We prove that positive solutions of (4) exist if relations in (5) hold and $b$ satisfiesy some additional assumptions. The main role in investigation performed below is played by the transcendental equation

$$
\lambda=a \exp \left(\lambda \sum_{i=1}^{n} \alpha_{i} \tau_{i}\right)
$$

We show that the transcendental equation (6) has two real roots and give its properties. The existence of a positive solution $y=y(t)$ of (4) is proved by utilization of a result on existence of solutions lying between two auxiliary functions. One of such functions is in the form of an exponential function constructed using a real root of (6). The paper is organized as follows. In subsection 2.1 essential auxiliary result on asymptotic behavior of solutions of general nonlinear system is cited for the reader's convenience (Theorem 1 below). Subsection 2.2 is devoted to the investigation of the properties of real roots of the transcendental equation (6). Then in section 3 auxiliary results are applied to the equation (4). For further results on the existence of positive solutions and also oscillating solutions to delayed differential equations we refer, e.g., to monographs [2] - [5], papers [1], [6] - [14] and to the references therein.

\subsection{A result based on the retract method}

Let $C\left([a, b], \mathbb{R}^{n}\right)$ where $a, b \in \mathbb{R}, a<b$, be the Banach space of the continuous mappings from the interval $[a, b]$ into $\mathbb{R}^{n}$ equipped with the supreme norm

$$
\|\phi\|_{c}=\sup _{\theta \in[a, b]}\|\phi(\theta)\|, \quad \phi \in C\left([a, b], \mathbb{R}^{n}\right)
$$

where $\|\cdot\|$ is the maximum norm in $\mathbb{R}^{n}$. In the case of $a=-\tau$ and $b=0$, we shall denote this space as $C_{\tau}^{n}$, that is,

$$
C_{\tau}^{n}:=C\left([-\tau, 0], \mathbb{R}^{n}\right)
$$

If $\sigma \in \mathbb{R}, A \geq 0$, and $y \in C\left([\sigma-\tau, \sigma+A], \mathbb{R}^{n}\right)$, then, for each $t \in[\sigma, \sigma+A]$, we define $y_{t} \in C_{\tau}^{n}$ by $y_{t}(\theta)=y(t+\theta), \theta \in[-\tau, 0]$.

We consider a system of retarded functional differential equations

$$
\dot{y}(t)=F\left(t, y_{t}\right)
$$

where $F: \Omega^{*} \mapsto \mathbb{R}^{n}, F=\left(F_{1}, \ldots, F_{n}\right)$ is a continuous quasibounded functional which satisfies a local Lipschitz condition with respect to the second argument and $\Omega^{*}$ is an open subset in $\mathbb{R} \times C_{\tau}^{n}$. We recall that the functional $F$ is quasi-bounded if $F$ is bounded on every set of the form $\left[t_{1}, t_{2}\right] \times C_{\tau L}^{n} \subset \Omega^{*}$, where $t_{1}<t_{2}, C_{\tau L}^{n}:=C([-\tau, 0], L)$ and $L$ is a closed bounded subset of $\mathbb{R}^{n}$ (compare [4, p. 305]). In accordance with [5] a function $y(t)$ is said to be a solution of system (7) on $[\sigma-\tau, \sigma+A)$ if there are $\sigma \in \mathbb{R}$ and $A>0$ such that $y \in C\left([\sigma-\tau, \sigma+A), \mathbb{R}^{n}\right),\left(t, y_{t}\right) \in \Omega^{*}$ and $y(t)$ satisfies the system (7) for $t \in[\sigma, \sigma+A)$. For given $(\sigma, \varphi) \in \Omega^{*}$, we say $y(\sigma, \varphi)$ is a solution of the system (7) through $(\sigma, \varphi)$ if there is an $A>0$ such that $y(\sigma, \varphi)$ is a solution of the system (7) on $[\sigma-\tau, \sigma+A)$ and $y_{\sigma}(\sigma, \varphi)=\varphi$. In view of above conditions each element $(\sigma, \varphi) \in \Omega^{*}$ determines a unique solution $y(\sigma, \varphi)$ of the system (7) through $(\sigma, \varphi) \in \Omega^{*}$ on its maximal interval of existence $I_{(\sigma, \varphi)}=[\sigma, a), \sigma<a \leq \infty$ which depends continuously on initial data ([5]). A solution $y(\sigma, \varphi)$ of the system (7) is said to be positive if

$$
y(\sigma, \varphi)>0
$$


on $[\sigma-\tau, \sigma] \cup I_{(\sigma, \varphi)}$ for each $i=1,2, \ldots, n$. If $I_{(\sigma, \varphi)}=[\sigma, \infty)$, then a nontrivial solution $y(\sigma, \varphi)$ of the system (7) is said to be oscillatory if (8) does not hold on any subinterval $\left[\sigma_{1}, \infty\right) \subset[\sigma, \infty), \sigma_{1} \geq \sigma$.

For continuous vector functions

$\rho=\left(\rho_{1}, \rho_{2}, \ldots, \rho_{n}\right), \delta=\left(\delta_{1}, \delta_{2}, \ldots, \delta_{n}\right):\left[t_{0}-\tau \infty\right) \rightarrow \mathbb{R}^{n}$

with $\rho(t) \ll \delta(t)$ for $t \in\left[t_{0}-\tau, \infty\right)$ (the symbol $\ll$ here and below means: $\rho_{i}(t)<\delta_{i}(t)$ for all $\left.i=1,2, \ldots, n\right)$, continuously differentiable on $\left[t_{0}, \infty\right)$, we define the set $\omega:=\left\{(t, y): t \in\left[t_{0}, \infty\right), \rho(t) \ll y \ll \delta(t)\right\}$.

In the sequel, we employ the following result being a particular case of [6, Theorem 1].

Theorem 1. Assume that if $t \geq t_{0}, \phi \in C_{\tau}^{n}$ and $(t+\theta, \phi(\theta)) \in \omega$ for any $\theta \in[-\tau, 0)$, then

$$
\begin{aligned}
& \delta_{i}^{\prime}(t)<F_{i}(t, \phi) \text { when } \phi_{i}(0)=\delta_{i}(t) \\
& \rho_{i}^{\prime}(t)<F_{i}(t, \phi) \text { when } \phi_{i}(0)=\rho_{i}(t)
\end{aligned}
$$

for any $i=1,2, \ldots, n$. Then there exists an uncountable set $\gamma$ of solutions of (7) on $\left[t_{0}-\tau, \infty\right)$ such that each $y \in \gamma$ satisfies

$$
\rho(t) \ll y(t) \ll \delta(t), t \in\left[t_{0}-\tau, \infty\right) .
$$

The original Theorem 1 is in [6] proved using the retract technique combined with Razumikhin's type ideas, known in theory of stability of retarded functional differential equations.

\subsection{Real roots of a transcendental equation}

Let us consider the auxiliary transcendental equation (6) In the following lemma we prove some properties of its real solutions.

Lemma 1. Let $a, \tau_{1}$ and $\alpha_{i}, i=1,2, \ldots, n$ be positive constants, and inequality (5) holds. Then there exist just two real different positive roots $\lambda_{1}, \lambda_{2}$ of (6) such that $\lambda_{1}<\lambda_{2}$. Moreover.
a) $\lambda_{1} \sum_{i=1}^{n} \alpha_{i} \tau_{i}<1$
b) $\lambda_{2} \sum_{i=1}^{n} \alpha_{i} \tau_{i}>1$

Proof. Let us define the auxiliary function

$$
f(\lambda):=\lambda-a \exp \left(\lambda \sum_{i=1}^{n} \alpha_{i} \tau_{i}\right)
$$

Looking for its extremal points, we compute its first derivative:

$$
f^{\prime}(\lambda):=1-a \sum_{i=1}^{n} \alpha_{i} \tau_{i} e^{\lambda \sum_{i=1}^{n} \alpha_{i} \tau_{i}}
$$

Equation $f^{\prime}(\lambda)=0$ has a root

$$
\lambda=\lambda_{\in}=-\frac{1}{\sum_{i=1}^{n} \alpha_{i} \tau_{i}} \ln \left(a \sum_{i=1}^{n} \alpha_{i} \tau_{i}\right)
$$

Since the second derivative $f^{\prime \prime}(\lambda)$ is a negative function, it is obvious that at $\lambda_{\in}$ the auxiliary function $f(\lambda)$ reaches its maximum. We compute it and conclude

$$
f\left(\lambda_{\epsilon}\right)=-\frac{1}{\sum_{i=1}^{n} \alpha_{i} \tau_{i}}\left[\ln \left(a \sum_{i=1}^{n} \alpha_{i} \tau_{i}\right)+1\right]>0
$$

if inequality (5) holds. Since $f(0)=-a<0$ and $f(+\infty)=-\infty$, the equation (6) has just two different positive roots $\lambda_{1}, \lambda_{2}$ satisfying $\lambda_{1}<\lambda_{\epsilon}<\lambda_{2}$. To prove the part $a$ ) we consider:

$$
\begin{aligned}
& \lambda_{1}=a \exp \left(\lambda_{1} \sum_{i=1}^{n} \alpha_{i} \tau_{i}\right)<a \exp \left(\lambda_{\epsilon} \sum_{i=1}^{n} \alpha_{i} \tau_{i}\right) \\
& =a \exp \left(-\frac{1}{\sum_{i=1}^{n} \alpha_{i} \tau_{i}} \ln \left(a \sum_{i=1}^{n} \alpha_{i} \tau_{i}\right) \sum_{i=1}^{n} \alpha_{i} \tau_{i}\right) \\
& =a\left(\frac{1}{a \sum_{i=1}^{n} \alpha_{i} \tau_{i}}\right)=\frac{1}{\sum_{i=1}^{n} \alpha_{i} \tau_{i}} .
\end{aligned}
$$

From this we have $\lambda_{1} \sum_{i=1}^{n} \alpha_{i} \tau_{i}<1$. Since $\lambda_{2}>\lambda_{\epsilon}$, part $b$ ) holds by the similar arguments. The lemma is proved.

In the proof of the main result we will need estimation of the expression

$$
g(\varepsilon):=\lambda_{2}\left(\varepsilon-\frac{a}{\lambda_{2}}\left(\frac{\lambda_{2}}{a}\right)^{\varepsilon}\right)-b(t)\left(\frac{\lambda_{2}}{a}\right)^{\varepsilon}
$$

where $\varepsilon$ is a constant. The following Lemma gives us a condition under that expression (12) is positive.

Lemma 2. If for function inequality

$$
b(t)<a\left(\left(\ln \frac{\lambda_{2}}{a}-\ln \left(\ln \frac{\lambda_{2}}{a}\right)-1\right)\right), t \in\left[t_{0}, \infty\right)
$$

holds, then $g(\varepsilon)>0$.

Proof. Let us define the auxiliary function

$$
f(\varepsilon)=\varepsilon-\frac{a}{\lambda_{2}}\left(\frac{\lambda_{2}}{a}\right)
$$

looking for its extremal point we compute its first derivative

$$
f^{\prime}(\varepsilon)=1-\frac{a}{\lambda_{2}}\left(\frac{\lambda_{2}}{a}\right)^{\varepsilon} \ln \frac{\lambda_{2}}{a} .
$$


Since the second derivative $f^{\prime \prime}(\varepsilon)$ is negative, function $f(\varepsilon)$ reaches in its extremal point $\varepsilon^{*}$ maximum. To find $\varepsilon^{*}$ we have to solve equation $f^{\prime}(\varepsilon)=0$. We get

$$
\left(\frac{\lambda_{2}}{a}\right)^{\varepsilon}=\frac{\lambda_{2}}{a} \frac{1}{\ln \frac{\lambda_{2}}{a}}
$$

and after necessary computations we have

$$
\varepsilon^{*}=1-\frac{\ln \left(\ln \frac{\lambda_{2}}{a}\right)}{\ln \frac{\lambda_{2}}{a}} .
$$

For clarity of next computations we use the substitution

$$
\ln \frac{\lambda_{2}}{a}=s, \text { i.e., } \frac{\lambda_{2}}{a}=e^{s} \text {. }
$$

From (6) and property $b$ ) in Lemma 1 it is visible that $s>1$. Now substituting $\mathcal{E}^{*}$ to (12) and using (15) and (17) we get

$$
g\left(\varepsilon^{*}\right)=g\left(1-\frac{\ln s}{s}\right)=\lambda_{2}\left(1-\frac{\ln s}{s}-\frac{1}{s}\right)-b(t) \frac{e^{s}}{s} .
$$

For the positivity of previous expression function $b(t)$ must fulfill the following inequality

$b(t)<e^{-s} \lambda_{2}(s-\ln s-1)$.

Using (17) we have (13). The lemma is proved

\section{Main result}

In this section we prove the existence of positive solutions to equation (4) and give its estimation from above.

Theorem 2. Let $b:\left[t_{0}, \infty\right) \rightarrow \mathbb{R},|b(t)|<a, t \in\left[t_{0}, \infty\right)$ and for (13) be fulfilled. Then equation (4) admits an uncountable set $\gamma$ of positive solutions defined on $\left[t_{0}-\tau, \infty\right)$ such that each $y=y(t) \in \gamma$ satisfies inequalities

$$
0<y(t)<e^{-\lambda_{2 t \varepsilon^{*}}}
$$

where is defined in Lemma 1 and is defined by (16).

Proof. To prove the theorem, we employ Theorem 1 with $n=1$,
$F\left(t, y_{t}\right):=-(a+b(t)) \prod_{i=1}^{n} y^{\alpha_{i}}\left(t-\tau_{i}\right)$,

$\rho(t):=0, \delta(t):=e^{\lambda_{2} t \varepsilon^{*}}$.

Hence the set is defined as

$\omega=\left\{(t, y): t \in\left[t_{0}-\tau, \infty\right), 0<y(t)<e^{\lambda_{2 t \varepsilon^{*}}}\right\}$.

Now, we have to verify the inequalities (9) and (10). In our case (for $\left.t \geq t_{0}\right)$

$F(t, \phi)-\delta^{\prime}(t)=-(a+b(t)) \prod_{i=1}^{n} y^{\alpha_{i}}\left(t-\tau_{i}\right)-\delta^{\prime}(t)$

$=-(a+b(t)) \prod_{i=1}^{n} y^{\alpha_{i}}\left(t-\tau_{i}\right)+\lambda_{2} \varepsilon^{*} e^{-\lambda_{2} t \varepsilon^{*}}$

$>-(a+b(t)) \prod_{i=1}^{n} \delta^{\alpha_{i}}\left(t-\tau_{i}\right)+\lambda_{2} \varepsilon^{*} e^{-\lambda_{2} t \varepsilon^{*}}$

$=-(a+b(t)) \exp \left(-\lambda_{2} \varepsilon^{*} \sum_{i=1}^{n} \alpha_{i}\left(t-\tau_{i}\right)\right)+\lambda_{2} \varepsilon^{*} e^{-\lambda_{2} t \varepsilon^{*}}$

$=e^{-\lambda_{2} t \varepsilon^{*}}\left[\lambda_{2} \varepsilon^{*}-(a+b(t)) \exp \left(\lambda_{2} \varepsilon^{*} \sum_{i=1}^{n} \alpha_{i} \tau_{i}\right)\right]$

$=\left(\mathrm{u} \sin \mathrm{g} \exp \left(\lambda_{2} \varepsilon^{*} \sum_{i=1}^{n} \alpha_{i} \tau_{i}\right)=\left(\frac{\lambda_{2}}{a}\right)^{\varepsilon^{*}}\right)$

$=e^{-\lambda_{2} t \varepsilon^{*}}\left[\lambda_{2} \varepsilon^{*}-a^{1-\varepsilon^{*}} \lambda_{2}^{\varepsilon^{*}}-b(t)\left(\frac{\lambda_{2}}{a}\right)^{\varepsilon^{*}}\right]$

$=e^{-\lambda_{2} t \varepsilon^{*}}\left[\lambda_{2}\left(\varepsilon^{*}-\frac{a}{\lambda_{2}}\left(\frac{\lambda_{2}}{a}\right)^{\varepsilon^{*}}\right)-b(t)\left(\frac{\lambda_{2}}{a}\right)^{\varepsilon^{*}}\right]$

$>0$ (due to Lemma2).

Hence the inequality (9) holds for $t \in\left[t_{0}, \infty\right)$. To verify the inequality (10) we have

$F(t, \phi)-\rho^{\prime}(t)=-(a+b(t)) \prod_{i=1}^{n} y^{\alpha_{i}}\left(t-\tau_{i}\right)-\rho^{\prime}(t)$

$=-(a+b(t)) \prod_{i=1}^{n} y^{\alpha_{i}}\left(t-\tau_{i}\right)<0$.

Since both inequalities are fulfilled and all assumptions of Theorem 1 are satisfied, the conclusion of the theorem is straightforward of the inequalities (11).

Corollary 1. If $y=y(t)$ is a solution to eq. (4) then also $y=C(t)$, where $C$ is a constant, is a solution to eq. (4).

Remark. For positive solutions satisfying (18) it holds: $\lim _{t \rightarrow \infty} y(t)=0$ This follows from that number $\varepsilon^{*}$ defined by (16) is positive due to the property b) in Lemma 1.

\section{References}

[1] DIBLIK, J., RUZICKOVA, M.: Asymptotic Behavior of Solutions and Positive Solutions of Differential Delayed Equations, Funct. Differ. Equ. 14, No. 1, 2007, 83-105.

[2] AGARWAL, R. P., BEREZANSKY, L., BRAVERMAN, E., DOMOSHNiTSKY, A.: Nonoscillation Theory of Functional Differential Equations with Applications, Springer, 2012. 
[3] AGARWAL, R. P., BOHNER, M., WAN-TONG, LI.: Nonoscillation and Oscillation: Theory for Functional Differential Equations, Marcel Dekker, Inc. 2004.

[4] DRIVER, R. D.: Ordinary and Delay Differential Equations, Springer-Verlag, 1977.

[5] HALE, J. K., LUNEL, S. M. V.: Introduction to Functional Differential Equations, Springer-Verlag, 1993.

[6] DIBLIK, J.: A Criterion for Existence of Positive Solutions of Systems of Retarded Functional Differential Equations, Nonlinear Anal., 38, 1999, 327-339.

[7] DIBLIK, J., KOKSCH, N.: Positive Solutions of the Equation in the Critical Case, J. Math. Anal. Appl., 250, 2000, 635-659.

[8] DIBLIK, J., KUDELCIKOVA, M.: Existence and Asymptotic Behavior of Positive Solutions of Functional Differential Equations of Delayed Type, Abstr. and Appl. Anal., vol. 2011, 2011, 16 p.

[9] DIBLIK, J., RUZICKOVA, M, SMARDA, Z.: Wazewski's Method for Systems of Dynamic Equations on Time Scales, Nonlinear Anal., 71, 2009, E1124-E1131. [10] Diвliк, J., Svoboda, Z.: An Existence Criterion of Positive Solutions of p-type Retarded Functional Differential Equations, J. Comput. Appl. Math. 147, 2002, 315-331.

[11] DIBLIK J., SVOBODA Z.: Positive Solutions of Retarded Functional Differential Equations, Nonlinear Anal., 63, $2005,813-821$.

[12] DIBLIK, J., SVOBODA, Z.: Positive Solutions of type Retarded Functional Differential Equations, Nonlinear Anal., 64, 2006, 1831-1848.

[13] DOMOSHNITSKY, A., DRAKHLIN, M.: Nonoscillation of First Order Differential Equations with Delay, J. Math. Anal. Appl., 206, 1997, 254-269.

[14] SAMAJOVA, H., SPANIKOVA, E., Dix, J. G.: Decay of Non-oscillatory Solutions for a System of Neutral Differential Equations, Electron. J. Differential Equations, No 271, 2013, 2013, 1-11. 